\title{
OLEOGELS OF OLIVE OIL AND SOYBEAN OIL FOR TOPICAL DRUG DELIVERY: A COMPARATIVE ANALYSIS
}

\author{
DIPANJANA ASH*, SUTAPA BISWAS MAJEE, GOPA ROY BISWAS
}

Department of Pharmacy, NSHM Knowledge Campus, Kolkata-Group of Institutions, 124 B L Saha Road, Kolkata 700053

Email: dipanjanaash16@gmail.com

Received: 10 May 2019 Revised and Accepted: 21 Jun 2019

\section{ABSTRACT}

Objective: The objective of the present investigation was to develop olive and soybean oil-based oleogels with Span 40 and/or Tween 80 (as gelator and/or surfactant) and determine the critical gelator concentration (CGC), characterise and compare the rheological, thermal properties and drug release profile of the gels formed for topical delivery.

Methods: Olive and soybean oil-based Span 40 and Span 40/Tween 80 oleogel formulations were prepared by solid fiber mechanism and subjected to organoleptic evaluation, FT-IR spectroscopy, thermal analysis, rheological study, kinetic modeling of gelation and drug release.

Results: The critical gelator (Span 40) concentration was found to be lower for olive oil (12\% w/v) and depend on the type of oil. Tween 80 reduced CGC of soybean oleogels only. Soybean oil-based oleogel containing $18 \% \mathrm{w} / \mathrm{v}$ Span 40 was found to form more flexible, less viscous and thermally less stable formulation with better release of paracetamol as evident from lower melt flow index, $\mathrm{T}_{\mathrm{g}}$ value, lower $\beta$ and higher $\alpha$ value compared to olive oil-based oleogel with $12 \% \mathrm{w} / \mathrm{v}$ Span 40 (CGC). Surfactant addition can be assumed to modify the microarchitecture of the oleogels to a great extent to produce more flexible and thermally stable gels with even better drug release profile. Span-Tween based soybean oleogel formed a gel-matrix whereas matrix in olive oil-based oleogels containing Span only became slightly flexible to release the drug in zeroorder fashion on the addition of surfactant cogelator.

Conclusion: Nature of oil exerts profound influence on the rheological, thermal and release profile of oleogels containing Span 40 as gelator and/or Tween 80 as surfactant cogelator.

Keywords: Accelerated thermal stability, Cogelator, Critical gelator concentration, Gel-matrix, Gel-sol transition temperature, Melt flow index, Span 40, Tween 80

(C) 2019 The Authors. Published by Innovare Academic Sciences Pvt Ltd. This is an open-access article under the CC BY license (http://creativecommons.org/licenses/by/4.0/) DOI: http://dx.doi.org/10.22159/ijpps.2019v11i8.34040

\section{INTRODUCTION}

Oleogels are semi-solid, non-crystalline, thermo-reversible viscoelastic systems, in which an external apolar phase gets immobilized within the spaces of the three-dimensional networked structure formed via physical interactions amongst the selfassembled structures of organogelators. They are resistant to the effects of moisture and do not require the addition of stabilizers or preservatives and hence possess an edge as a drug delivery system over conventional gels. They are preferable for topical application owing to their ability to spread evenly as a film over the surface of the skin $[1,2]$. An ideal oleogel for the topical application would be one with good organoleptic properties, satisfactory extrudability and spreadability, high flexibility, high thermal stability and improved drug release.

Sterol, sorbitan monostearate (Span 60), sorbitan mono palmitate (Span 40), and cholesteryl anthraquinone derivates have been used as organogelators in the development of oleogels with different apolar solvents such as organic solvents (cyclohexane, benzene and carbon tetrachloride), vegetable oils (sunflower oil, rice bran oil, sesame oil etc.) and mineral oil [3-5]. In the development of oleogels Tween 20, Tween 80 which are primarily surfactant may be employed as co-gelator to obtain better performance, improved entrapment efficiency, solubility and drug permeation as evident from the various studies on topical microemulsion gel, emulgel and floating gastric nano-emulsion in-situ gel [6-8]. Tween 80 is reported to induce gelation for Span 80 based oleogels. There are also reports of organogels where an aqueous phase has been added to induce gelation [9]. For each apolar solventorganogelator/cogelator pair, there exists a unique critical gelator concentration (CGC) which is defined as the minimum concentration of gelator and gelator/cogelator required to induce gelation for particular oil [4].
Olive oil and soybean oil are suitable for topical applications as they provide nourishment to the skin. Soybean oil containing gels and lotions reportedly protects our skin from UVB rays, free radicalinduced inflammation, reduce transdermal water loss on the skin and promote skin barrier recovery [10]. No previous attempts have been made to utilize minimum concentration of Span 40 as an organogelator individually or in combination with Tween 80 as a surfactant/cogelator in the development of soybean oil and olive oilbased oleogels for topical drug delivery.

The objective of present investigation is to develop oleogels of soybean oil and olive oil with Span 40 or Span 40-Tween 80 combination, to determine CGC of either Span 40 alone or in combination with Tween 80 for both oils and to characterise and analyse the gels at their CGCs for organoleptic characteristics, thermal and rheological behaviour, drug release profile and establish their suitability for topical applications.

\section{MATERIALS AND METHODS}

\section{Materials}

Soybean oil (Emami Ltd., India) and olive oil (Park Daniel, India) were procured from the local market, Kolkata, West Bengal. Span 40 and Tween 80 were of AR grade and obtained from Loba Chemie and Merck Specialities Pvt. Ltd respectively and paracetamol IP (PCM) was received as a gift sample from the enlisted vendor. Double-distilled water was used throughout the study, wherever required. For hemocompatibility study, fresh goat blood was collected in the heparin-coated tube and stored at $-4^{\circ} \mathrm{C}$.

\section{Methods}

Accurately weighed Span 40, paracetamol (PCM) (2\% w/v for drug-loaded batches) and/or Tween 80 were dissolved in 
soybean oil/olive oil and temperature maintained at $60 / 70^{\circ} \mathrm{C}$ respectively. Stirring was continued at 500-1000 r. p. m depending on the oil to obtain a clear, homogeneous solution. Subsequent cooling down to $25^{\circ} \mathrm{C}$ formed an oleogel on gelation.
The formulations were stored in glass vials and considered to be gel if they did not flow on inversion. Oleogels of two oils were prepared with or without surfactant according to the composition given in table 1.

Table 1: Composition of oleogels

\begin{tabular}{|c|c|c|c|c|c|}
\hline \multirow[t]{3}{*}{ Batch } & \multicolumn{5}{|c|}{ Components (\%w/v) } \\
\hline & \multirow[t]{2}{*}{ Span 40} & \multirow[t]{2}{*}{ Tween 80} & \multicolumn{2}{|l|}{ Oil } & \multirow[t]{2}{*}{ PCM } \\
\hline & & & Soybean oil & Olive oil & \\
\hline OGS & 16 & - & 82 & - & 2 \\
\hline OGS 1 & 18 & - & 80 & - & 2 \\
\hline OGS 2 & 20 & - & 78 & - & 2 \\
\hline OGST 1 & 10.67 & 5.33 & 82 & - & 2 \\
\hline OGST 2 & 12 & 6 & 80 & - & 2 \\
\hline OGST 3 & 13.33 & 6.67 & 78 & - & 2 \\
\hline OGO & 10 & - & - & 88 & 2 \\
\hline OGO 1 & 12 & - & - & 86 & 2 \\
\hline OGO 2 & 14 & - & - & 84 & 2 \\
\hline OGOT & 6.67 & 3.33 & - & 88 & 2 \\
\hline OGOT 1 & 8 & 4 & - & 86 & 2 \\
\hline OGOT 2 & 9.33 & 4.67 & - & 84 & 2 \\
\hline
\end{tabular}

OGS* 1, OGST*1, OGO* 1 , OGOT* 1 are the blank oleogels of their corresponding drug-loaded oleogels and used for all investigations except drug release studies

\section{Evaluation of oleogels}

\section{Fourier transform infrared (FT-IR) spectroscopy}

Infrared spectroscopy of raw materials, blank and drug-loaded oleogels were scanned by using FT-IR spectroscopy(Bruker, Alpha$\mathrm{T}$ ) in attenuated total reflectance (ATR) mode in the range of 4000$500 \mathrm{~cm}^{-1}$.

\section{CGC determination}

The minimum concentration $(\% \mathrm{w} / \mathrm{v})$ of gelator/gelator-surfactant required to form gel is considered as critical gelator concentration for a particular oil.

\section{Organoleptic evaluation}

The freshly prepared blank gel formulations (OGS* 1, OGST* $^{*} 1$, OGO* 1 and OGOT* 1 ) were subjected to organoleptic evaluation for their color, odour, opacity and appearance.

\section{Applicability parameters}

Applicability parameters of oleogels include determination of extrudability and spreadability. Extrusion of oleogel was studied by measuring the distance travelled by the ribbon of gel extruded from a collapsible tube in $10 \mathrm{~s}$. Approximately $1 \mathrm{~g}$ of the oleogel was placed between two glass slides of equal weight, area and thickness. Initial spreading diameter $\left(D_{i}\right)$ was noted. Thereafter, a load of a known weight of $10,20,50$, or $100 \mathrm{~g}$ was applied individually on the upper slide for $1 \mathrm{~min}$ and the final spreading diameter $\left(\mathrm{D}_{\mathrm{f}}\right)$ of the gel was noted in each case. Extrudibilityand spreadability are expressed in $\mathrm{cm} / \mathrm{s}$ and percentage respectively. The $\%$ spreadability was calculated as per the equation is given below [5].

$$
\% \text { Spreadibility }=[(\mathrm{Df}-\mathrm{Di}) \div \mathrm{Di}] \times 100
$$

\section{pH measurement}

The $\mathrm{pH}$ was measured by immersing the glass electrode of the digital $\mathrm{pH}$ meter (EUTECH INSTRUMENTS pH Tutor) in the prepared drugloaded oleogel.

\section{Drug content study}

A definite amount of drug-loaded oleogels was mixed with phosphate buffer ( $\mathrm{pH}$ 5.8) to obtain uniform dispersion that was kept undisturbed for $48 \mathrm{~h}$ and was filtered through Whatman filter paper (No.1). An aliquot of the filtrate was suitably diluted and analysed by
UV-visible spectrophotometer (UV 1800 UV-vis spectrophotometer, Shimadzu Corporation) at a wavelength of $249 \mathrm{~nm}$ [11-12].

\section{Thermal analysis}

\section{a. Gelation kinetics}

Gelation kinetics was performed by nepheloturbidometry ((ELICO ${ }^{\circledR}$ CL 52D). Oleogel in sol state was transferred to Nessler cylinder. When the light passes through the sample having turbidity, light is scattered by the suspended particles. The scattering of light is proportional to the turbidity. Turbidity was measured at $20 \mathrm{~s}$ interval and expressed in terms of the nepheloturbidity unit (NTU). Transformation of sol to gel was characterized by an increase in turbidity, which continued for a certain period of time after which there was no further increase in turbidity. The time at which turbidity attained a constant value is defined as gelation time.

\section{b. Gelation kinetics modeling}

Gompertz model was employed for modelling of gelation kinetics. This non-linear model (equation 2) indicates a relationship between turbidity intensity (NTU)(Y), the concentration of gelator or gelator-surfactant in $\% \mathrm{w} / \mathrm{v}(\rho)$ and time for gelation in $\mathrm{h}(\mathrm{x})$.

$\alpha$ is defined as apolar solvent (oil) parameter, whereas $\beta$ indicates organogelator parameter.

$$
\log Y=\alpha+\beta \rho^{x}
$$

In the above equation, $\rho^{\mathrm{x}}$ is defined as

$$
\rho^{\mathrm{x}}=\rho_{1}{ }^{\mathrm{x}}+\rho_{2}{ }^{\mathrm{x}}+\rho_{3}{ }^{\mathrm{x}}+
$$

Where $\rho_{1}$ indicates organogelator concentration, $\rho_{2}$ indicates cogelator concentration; $\rho_{3}$ indicates other additive concentration and so on [13].

\section{c. Determination of Gel-sol transition temperature $\left(\mathrm{T}_{\mathrm{g}}\right)$}

Thermal analysis of oleogel was done by a drop ball method for determination of the gel-sol transition temperature $\left(\mathrm{T}_{\mathrm{g}}\right)$. A stainless steel ball having the diameter of $1 / 8^{\text {th }}$ inch and a weight of $230 \mathrm{mg}$ was placed over the formulation in a beaker and attached with a melting point apparatus (EI-931). The formulation was heated at a rate of $1^{\circ} \mathrm{C} / \mathrm{min}$. The temperature at which the ball started to move into the gel was noted and considered as the gel-sol transition temperature of gel $\left(\mathrm{T}_{\mathrm{g}}\right)[4]$. 


\section{d. Determination of melt flow index}

Melt flow index (MFI) is defined as the ease of melt flow of thermoplastic material in gram over a period of $10 \mathrm{~min}$ at a certain standard temperature (i. e, $65^{\circ} \mathrm{C}$ for gel formulations) when checked in melt flow tester. A fixed weight of oleogel formed (10 g) was poured into the cylinder and temperature was set at $65^{\circ} \mathrm{C}$ to prevent thermal degradation of oleogel at higher temperature as specified in the reported method. Pressure was applied with the piston bar to the cylinder using $10 \mathrm{~g}$ of weight above the piston bar after setting the predefined temperature. Sample flow occurred through the die face in the form of wire and was collected after $10 \mathrm{~min}$ and was further subjected to weighing to determine its mass [14].

\section{Rheological study}

Rheological study was performed by determining the viscosities of OGS* 1, OGST* $^{*} 1$, OGO* $^{*} 1$ and OGOT* 1 in Brookfield digital viscometer (Model LVDVI+) at $25^{\circ} \mathrm{C}$. The study was done by applying a shear rate of 1-5 r. p. m (spindle 6) for 1 min. Ostwald de-wale power model (equation 4) was employed to establish a relationship between shear rate $(\gamma)$, shear stress (T), flow behavior index (n) and flow consistency index $(\mathrm{k})$ [3].

$$
\tau=\kappa \times \gamma n_{\text {. }}
$$

\section{In vitro drug release study}

Modified Franz diffusion cell was used to perform the in vitro drug release study on oleogels at their CGC through dialysis membrane-60 (HIMEDIA ${ }^{\circledR}$ LA 330-5MT). Accurately weighed drug-loaded oleogel containing PCM equivalent to $4 \mathrm{mg}$ was placed on the membrane and wetted slightly with phosphate buffer (pH5.8). The buffer solution in the receptor compartment was maintained at $32 \pm 0.5^{\circ} \mathrm{C}$. An aliquot of $1 \mathrm{ml}$ was withdrawn every hour for $7 \mathrm{~h}$ and replenished with fresh buffer. Following appropriate dilution, aliquot was analyzed by UV-visible spectrophotometer (UV 1800 UV-vis spectrophotometer, Shimadzu Corporation) at a wavelength of $249 \mathrm{~nm}$. The release kinetics modelling was done from cumulative percent release (CPR) data [12].

\section{Determination of steady-state flux and permeability co-efficient}

The measurement of flux across human skin provides a valuable insight into the formulation development of any dermatological product. The steady-state flux of PCM from oleogels across the artificial dialysis membrane is defined as follows.

$$
\text { SSflux }=\frac{\mathrm{dQ}}{\mathrm{dt}} \times 1 \div \mathrm{A}
$$

Where,

$\mathrm{SS}_{\text {flux }}=$ steady-state flux of drug $\left(\mathrm{mg} / \mathrm{cm}^{2} . \mathrm{hr}\right) ; \mathrm{dQ} / \mathrm{dt}=$ slope of the linear portion of the curve i.e. cumulative amount per unit time $(\mathrm{mg} / \mathrm{hr}) ; \mathrm{A}=$ diffusional area $\left(\mathrm{cm}^{2}\right)$

Permeability co-efficient is quantified by the following equation.

$$
\mathrm{Kp}=\text { SSflux } \div \text { Capp }
$$

Where,

$\mathrm{C}_{\mathrm{app}}=$ initial concentration of the drug in the gel formulation. In the present study, it was expressed as \%w/v i.e. weight of drug actually present in the volume of gels taken for the permeation study [15].

\section{Hemocompatibility study}

Accurately weighed (1g) oleogel was placed inside dialysis tubing, immersed in $50 \mathrm{ml}$ of normal saline and incubated at $37^{\circ} \mathrm{C}$ for $1 \mathrm{~h}$ in a shaker incubator so as to allow the leaching of the components from the oleogels. A small volume $(0.5 \mathrm{ml})$ of the leachant was then diluted with $0.5 \mathrm{ml}$ of diluted goat blood (prepared by diluting $8 \mathrm{ml}$ of fresh goat blood with $10 \mathrm{ml}$ of normal saline) followed by the addition of $9 \mathrm{ml}$ of normal saline. The mixture was then incubated at $37^{\circ} \mathrm{C}$ for $1 \mathrm{~h}$ followed by centrifugation at $3000 \mathrm{rpm}$ for $10 \mathrm{~min}$. Positive and negative controls were also prepared by using $0.1 \mathrm{~N}$ hydrochloric acid and normal saline in place of the leachant, respectively. The supernatant was analysed at $545 \mathrm{~nm}$ using UVvisible spectrophotometry. The test measures the extent of haemolysis in the presence of the oleogel. Percent haemolysis is calculated by the formula [16].

$\%$ Hemocompatibility $=($ ODtest - ODnegative $) \div($ ODpositive - ODnegative $)$

Where,

$\mathrm{OD}_{\text {test }}=$ optical density of test sample, $\mathrm{OD}_{\text {negative }}=$ optical density of negative control, $\mathrm{OD}_{\text {positive }}=$ optical density of positive control.

\section{Accelerated stability study}

Accelerated stability study includes thermo-cycling or freeze/thaw cycling, and syneresis measurements to assess the change in gelation time with consecutive freeze-thaw cycles. Thermo-cycling or freeze-thaw cycling method involves incubation of the freshly prepared oleogels at $65^{\circ} \mathrm{C}$ for $15 \mathrm{~min}$ till the formation of sol state followed by gelation when the time was noted. Then these gel formulations were incubated at $4^{\circ} \mathrm{C}$ for $15 \mathrm{~min}$ after which stored at $25{ }^{\circ} \mathrm{C}$ for $48 \mathrm{hr}$. The cycle was repeated for 6 cycles for both soybean and olive oleogels [4], [16].

\section{Statistical analysis}

Data have been obtained from each experiment in triplicate $(n=3)$ and were subjected to statistical analysis using oneway analysis of variance (ANOVA). Results are quoted as significant where $\mathrm{p}<0.05$.

\section{RESULTS}

\section{Oleogel formation and CGC determination}

Oleogel formation was induced at different Span and Span-Tween concentration for soybean oil and olive oil. Gelation was induced at lower Span concentration in case of olive oil. CGC of Span 40 for olive oil based oleogels was found to be $12 \% \mathrm{w} / \mathrm{v}$ (OGO 1) whereas the CGC was $18 \%$ w/v (OGS 1) for soybean oil based gels. Addition of Tween 80 as surfactant in the ratio of Span: Tween at 2: 1 lowered the CGC for only soybean oil to $16 \% \mathrm{w} / \mathrm{v}$ (OGST 1 ). There was no change in CGC for olive oil by addition of Tween. Span: Tween at 2: 1 formed olive oil gel (OGOT 1) where the gelator-surfactant concentration was $12 \% \mathrm{w} / \mathrm{v}$. Span 40 : Tween 80 in the ratio of $1: 1$ or 1:2 did not cause gel formation in either of the cases (not reported here).

\section{Fourier Transform Infrared (FT-IR) spectroscopy}

The FT-IR profile is presented in (fig. 1). Peaks at 2913, 2855, 1744, 1157 and $719 \mathrm{~cm}^{-1}$ were observed in the spectrum of soybean oil whereas olive oil showed peaks at 2923, 2854, 1744, 1458, 1158 and $720 \mathrm{~cm}^{-1}$. Most of the characteristic peaks of Span 40, Tween 80 , soybean oil/olive oil, and paracetamol were observed in OGS 1 , OGO 1, OGST 1 and OGOT 1.

\section{Organoleptic and applicability parameters evaluation}

The organoleptic and applicability parameters of OGS* 1 , OGO* 1 , OGST* 1 and OGOT* 1 were found to be satisfactory (table 2 ).

\begin{tabular}{|c|c|c|c|c|c|c|}
\hline \multirow[t]{2}{*}{ Batch } & \multicolumn{4}{|c|}{ Organoleptic evaluation } & \multicolumn{2}{|c|}{ Applicability parameters } \\
\hline & Colour & Odour & Opacity & Appearance & Extrudi-bility $(\mathrm{cm} / \mathrm{s})$ & $\%$ Spreadability at different loads \\
\hline OGS*1 & Yellowish-white & Odourless & Opaque & Smooth-oily & $0.8 \pm 0.6$ & $10-78$ \\
\hline OGST* $^{*}$ & Yellow & Odourless & Transparent & Smooth-oily & $1.2 \pm 0.5$ & $12-50$ \\
\hline OGO* 1 & Whitish-yellow & Odourless & Opaque & Smooth-oily & $1.1 \pm 0.7$ & $10-70$ \\
\hline OGOT* 1 & Whitish-yellow & Odourless & Transparent & Smooth-oily & $2 \pm 0.8$ & $15-45$ \\
\hline
\end{tabular}

Table 2: Determination of organoleptic and applicability parameters of soybean oil-and olive oil-based oleogels at their CGCs. aData represent mean \pm standard deviation for 3 experiments $(n=3)$ 

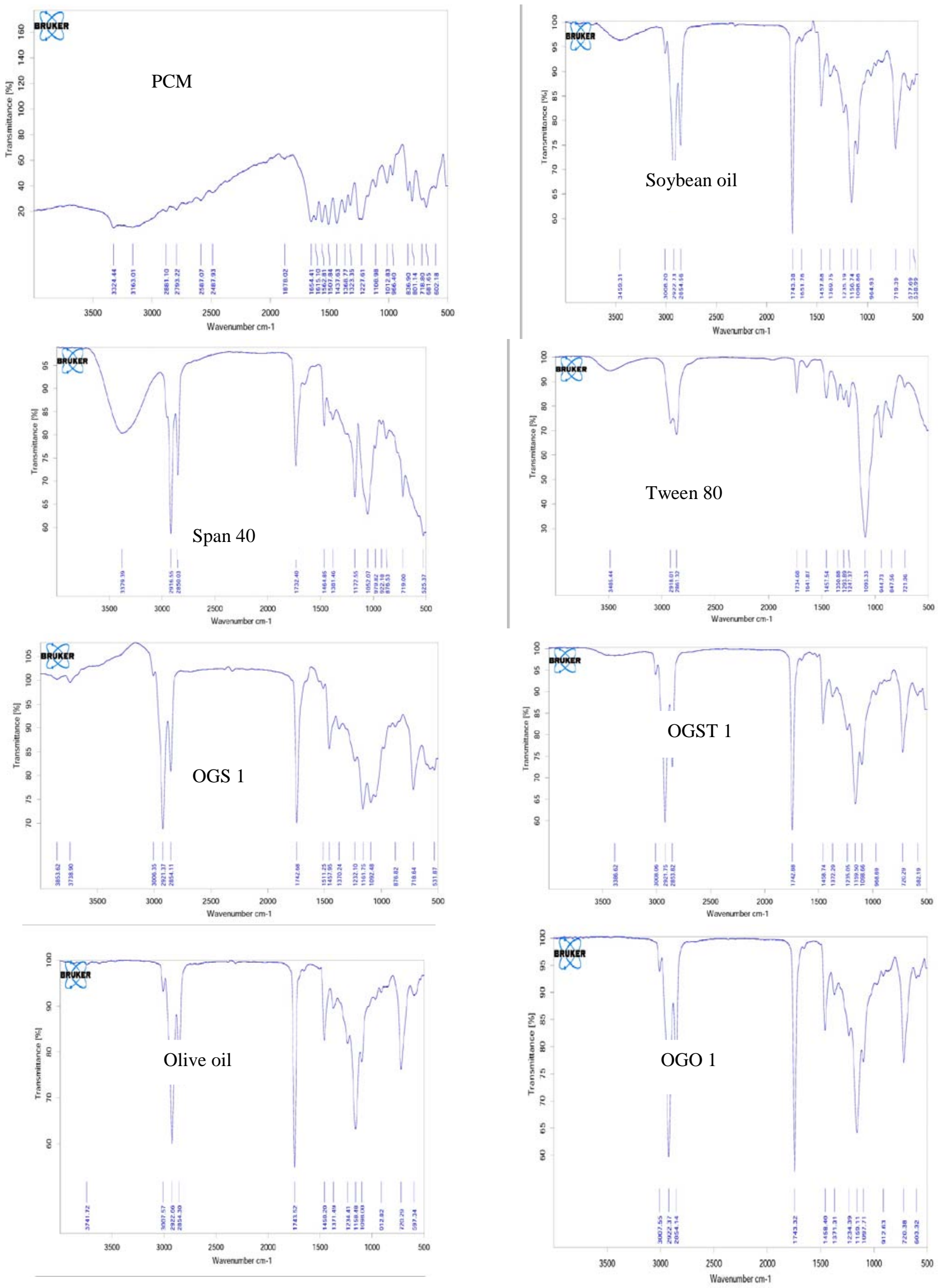


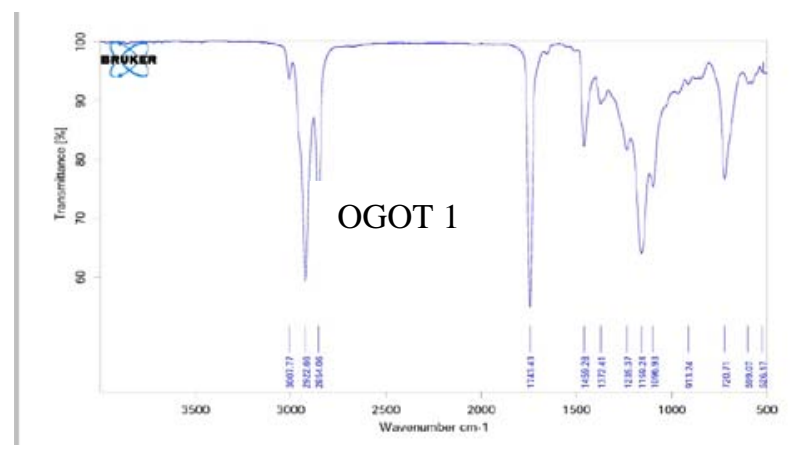

Fig. 1: FT-IR spectra of drug-loaded oleogels at their CGC along with raw materials

\section{pH measurement}

The gel formulations of soybean oil and olive oil were found to be compatible with skin $\mathrm{pH}(5.5 \pm 0.7)$ at $25^{\circ} \mathrm{C}$.

\section{Drug content study}

Drug content for all formulations was found to be in the range of 95$97 \%$.

\section{Thermal analysis}

Gelation time, gel-sol transition temperature, melt flow index and gelation kinetics modelling parameters [oil parameter $(\alpha)$ and organogelator parameter $(\beta)]$ of blank oleogels at their CGCs are presented in tabular form (table 3 ).

\section{Rheological study}

The order of viscosity of the four blank oleogel formulations at their CGCs is as follows: 0GO* $1>0$ GS $^{*} 1>0$ OGOT* $^{*} 1 \approx$ OGST* $^{*} 1$ Viscosity values are represented graphically (fig. 2). Oleogels showed pseudoplastic flow behaviour $(n<1)$, as evident from viscosity modelling.

\section{In vitro drug release study}

Highest drug release was obtained from oleogels of soybean oil and olive oil containing Tween as cogelator. However, paracetamol release was lower from olive oil-based gel. The CPR profiles of the gels are represented in fig. 3 . The kinetic model and mechanism of diffusion followed by oleogels are summarized in table 4 .

Table 3: Thermal analysis data of soybean oil-and olive oil-based oleogels at their CGCs. aData represent mean \pm standard deviation for 3 experiments $(n=3)$

\begin{tabular}{|c|c|c|c|c|c|}
\hline \multirow[t]{2}{*}{ Batch } & \multirow[t]{2}{*}{ Gelation time $(s)^{a}$} & \multirow[t]{2}{*}{ Gel-sol transition temperature $\left(\mathrm{T}_{\mathrm{g}}\right)\left({ }^{\circ} \mathrm{C}\right)^{\mathrm{a}}$} & \multirow[t]{2}{*}{ Melt flow index $(\mathrm{g} / 10 \mathrm{~min})^{a}$} & \multicolumn{2}{|c|}{ Modelling of gelation kinetics } \\
\hline & & & & $\alpha$ & $\beta$ \\
\hline $\mathrm{OGS}^{*} 1$ & $1060 \pm 1.2$ & $42 \pm 0.1$ & $0.87 \pm 0.6$ & 2.0726 & 0.5791 \\
\hline OGST* 1 & $1400 \pm 1.5$ & $49 \pm 0.2$ & $3.75 \pm 0.5$ & 2.5708 & 0.1799 \\
\hline OGO* 1 & $1100 \pm 1.3$ & $43 \pm 0.3$ & $0.34 \pm 0.4$ & 1.8907 & 0.656 \\
\hline OGOT $^{*} 1$ & $1440 \pm 1.6$ & $50 \pm 0.5$ & $2.88 \pm 0.6$ & 2.5702 & 0.1801 \\
\hline
\end{tabular}

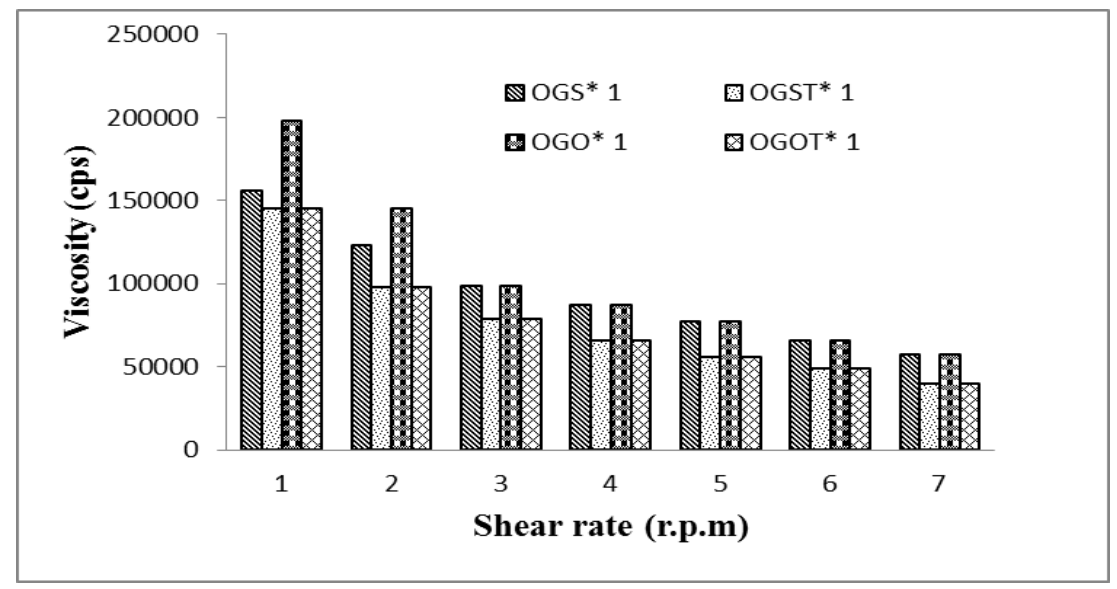

Fig. 2: Viscosity profile of soybean oil-and olive oil-based oleogels at their CGCs

Table 4: Release kinetic modelling of soybean oil-and olive oil-based oleogels at their CGCs

\begin{tabular}{lll}
\hline Batch & Kinetic followed & Mechanism of diffusion. \\
\hline OGS 1 & Zero & Fickian \\
OGST 1 & Higuchi & Not specifically defined \\
OGO 1 & Higuchi & Non-Fickian \\
OGOT 1 & Zero & Fickian \\
\hline
\end{tabular}




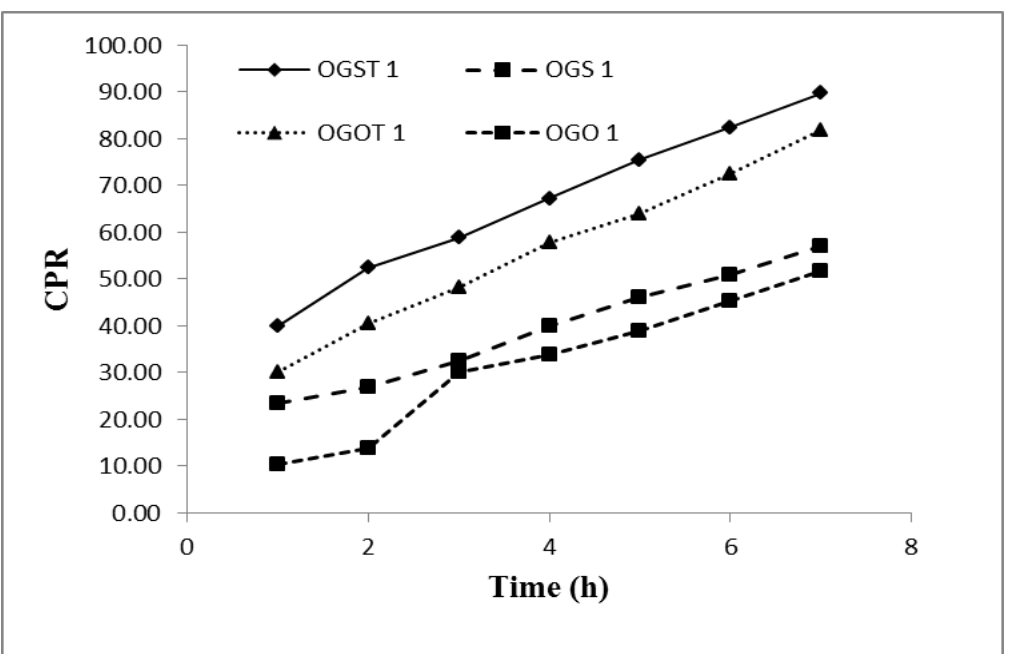

Fig. 3: CPR profile of soybean oil-and olive oil-based oleogels at their CGCs. Error bars represents mean \pm standard deviation for 3 experiments $(n=3)$

Table 5: Values of $t_{50}$, steady state flux and permeability co-efficient of oleogels, data represent mean \pm standard deviation for 3 experiments $(n=3)$

\begin{tabular}{llll}
\hline Batch & $\mathbf{t}_{\mathbf{5 0}} \mathbf{( h )}$ & Steady state flux $\mathbf{( m g} \mathbf{~ c m} / \mathbf{h})$ & Permeability co-efficient (cm/h) \\
\hline OGS 1 & $6.0 \pm 0.3$ & $2.68 \pm 0.5$ & $1.34 \pm 0.2$ \\
OGST 1 & $1.8 \pm 0.6$ & $2.81 \pm 0.2$ & $1.41 \pm 0.3$ \\
OGO 1 & $6.8 \pm 0.5$ & $1.72 \pm 0.4$ & $0.86 \pm 0.4$ \\
OGOT 1 & $3.2 \pm 0.5$ & $3.52 \pm 0.3$ & $1.76 \pm 0.8$ \\
\hline
\end{tabular}

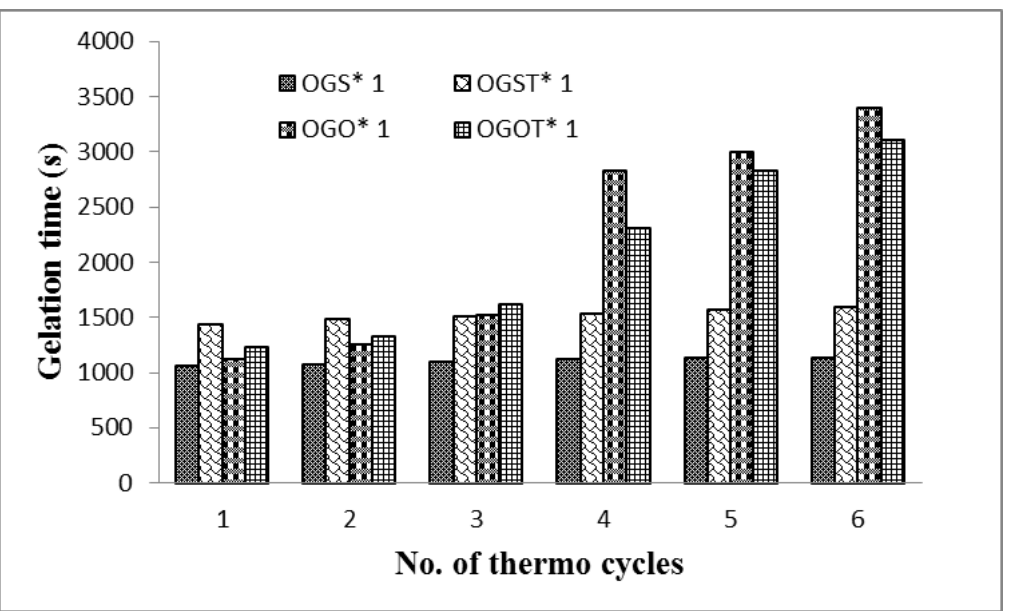

Fig. 4: Change in gelation time (s) with thermo cycles for oleogels of soybean oil and olive oil. Error bars represent mean \pm standard deviation for 3 experiments $(n=3)$

\section{Determination of steady-state flux and permeability co-efficient}

Steady-state flux, permeability co-efficient and $t_{50}$ values of oleogels at their CGCs are summarized in table 5.

\section{Hemocompatibility study}

The four formulations were found to be highly hemocompatible $(<5)$ [14].

\section{Accelerated stability study}

Soybean and olive oil-based oleogels at their CGC were found to be stable up to 6 thermo-cycles which are represented graphically (fig. 4).

\section{DISCUSSION}

The dissolution of Span 40 in the soybean oil and olive oil at 60 and $70{ }^{\circ} \mathrm{C}$ respectively resulted in the formation of clear homogeneous solution but change in solubility parameter of Span 40 molecules with lowering of temperature to $25^{\circ} \mathrm{C}$ decreased the affinity between continuous oil phase and Span and therefore a three dimensional self-assembled structure of gelator was formed by capturing oil molecules within [4]. The critical gelator (Span 40) concentration was found to depend on the type of oil. Tween 80 showed an effect only on CGC of soybean oleogels. Higher density of olive oil might have inhibited CGC lowering of the gels by addition of Tween 80 .

FTIR analysis of the oleogels revealed slight shifting in the peaks of the individual components as well as minor changes in peak intensity indicating compatibility between the oil, organogelator, surfactant and drug. Intermolecular hydrogen bonding amongst the fatty acyl groups of gelator and oil molecules, which is responsible for imparting strength to the gels due to the absence of the broad peak at $3300 \mathrm{~cm}^{-1}$ in oleogels with or without Tween 80 [17-18]. 
No variations in organoleptic properties were observed in soybean and olive oil-based oleogels at their CGC except colour but surfactant addition changed the colour and enhanced the transparency of the oleogels.

All the formulations were found to possess satisfactory applicability parameters, skin-compatible $\mathrm{pH}$, hemocompatibility and nonNewtonian pseudo-plastic flow behavior indicating their suitability for topical applications.

Thermal analysis revealed that more time was required to induce gelation in OGO* 1 than with $\mathrm{OGS}^{*} 1$. Similar delay in gelation was observed after Tween addition for olive oil. Comparatively higher $\mathrm{T}_{\mathrm{g}}$ value, lower melt flow index, lower $\alpha$ value and higher $\beta$ value of OGO* 1 indicated its compactness, more rigidity and better thermal stability than OGS* 1 . It is noteworthy to mention that the addition of Tween produced oleogels of soybean oil and olive oil with identical thermal stability (similar values of $\mathrm{T}_{\mathrm{g}}$ and $\beta$ ), and flexibility (approximately equal $\alpha$ value). Thus, Tween 80 can be assumed to modify the microarchitecture of the oleogels to a great extent and produce flexible gels with expected better drug release parameters. Greater flexibility in soybean oil-based OGS 1 and OGST 1 is expected to produce better drug release compared to olive oil-based oleogels. In vitro drug release data and values of $\mathrm{SS}_{\text {flux }}$ and $\mathrm{Kp}$ revealed OGS 1 to be better than OGO 1 . The time taken to release $50 \%$ of the drug was similar for the two formulations, which is evident from the drug release profile curves. Drug release from OGS 1 followed zero-order kinetics with Fickian diffusion whereas OGO 1 is assumed to form rigid gel-matrix with comparatively lower values of $\mathrm{SS}_{\text {flux }}$ and $\mathrm{Kp}$ and followed Higuchi kinetics with non-Fickian diffusion. Shah et al. reported that olive oil-based Span 60 and Span 40 oleogel followed Higuchi model indicating planar homogeneous matrix nature of gels with no loss of structural integrity [4]. Drug release from matrix formulation (OGO 1) might have occurred by the gradual break-up of the gel matrix into smaller fragments as the gel skeleton is compromised by the influx of dissolution medium via the conduits offered by the tubular structure of gelator molecules. Finally, the drug-loaded oil droplets could be released [19].

Significant improvement in drug release was observed in both the oleogels of soybean oil and olive oil containing Tween 80 as cogelator. OGST 1 revealed comparatively better drug release than OGOT 1. Drug release from OGST 1 and OGOT 1 followed Higuchi and zero-order kinetics, respectively. Fickian diffusion was observed in the case of OGOT 1. Addition of surfactant (Tween 80) as co-agulator thus altered the release kinetics i, e, surfactant addition in soybean oleogels formed a gel matrix whereas matrix in olive oil-based oleogels became slightly flexible to release the drug in zero-order fashion when Tween was added.

Summing up the observations from thermal analysis and drug release studies on oleogels of soybean oil and olive oil with Span 40 as gelator and Tween 80 as co-gelator, it can be inferred that more flexible gels with better drug release properties but lower thermal stability can be obtained with soybean oil. Drug release can follow either the zero-order kinetics or Higuchi model depending on the presence of surfactant co-gelator.

From accelerated thermal stability study, it was found that all formulations were found to be stable up to 6 cycles, although there were a significant increase in gelation time from $4^{\text {th }}$ cycle onwards in case of olive oil-based oleogels. This might be attributed to too much rigidity in the gel microstructure which once broken during thawing took more time to build up or reform. In the case of soybean oilbased oleogels, flexibility imparted stability against complete disintegration of gel structure during repeated freeze-thaw cycles.

\section{CONCLUSION}

Soybean oil-based oleogels possess higher flexibility with better drug release properties and lower thermal stability, but oleogels of olive oil were formed at the lower concentration of gelator and gelator/co-gelator. Comparative analysis of oleogels of soybean oil and olive oil for topical drug delivery thus demonstrated the significant influence of the type of oil on the thermal properties, gel flexibility, drug release behaviour and thermal stability.

\section{AUTHORS CONTRIBUTIONS}

Authors contributed equally to the design and implementation of the research, to the analysis of the results and the writing of the manuscript.

\section{CONFLICTS OF INTERESTS}

Authors declare that they have no conflicts of interest

\section{REFERENCES}

1. Nigar KM, Sangramsinh LG, Veerendra CY. Organogel: factors and its importance. Int J Bio Chem Sci 2014;4:758-73.

2. Rehman K, Zulfakar MH. Recent advances in gel technologies for topical and transdermal drug delivery. Drug Dev Indian Pharm 2014;40:433-40.

3. Singh VK, Pramanik K, Ray SS, Pal K. Development and characterization of sorbitan monostearate and sesame oilbased organogels for topical delivery of antimicrobials. AAPS PharmSciTech 2015;16:293-305.

4. Shah DK, Sagiri SS, Behera B, Pal K, Pramanik K. Development of olive oil-based organogels using sorbitan monopalmitate and sorbitan monostearate: a comparative study. J Appl Poly Sci 2013;129:793-804.

5. Satapathy D, Sagiri SS, Pal K, Pramanik K. Development of mustard oil-and groundnut oil-based Span 40 organogels as matrices for controlled drug delivery. Des Monomers Polym 2014;17:545-56.

6. Iradhati $\mathrm{AH}$, Jufri M. Formulation and physical stability test of griseofulvin microemulsion gel. Int J Appl Pharm 2017;9:23-6.

7. Daood NM, Jassim ZE, Gareeb MM, Zeki H. Studying the effect of different gelling agent on the preparation and characterization of metronidazole as topical emulgel. Asian J Pharm Clin Res 2019;12:571-7.

8. Almajidi YQ, Albaderi AA, Fadhel H. Enhance solubility and prolong release of prochlorperazine maleate using floating nanoemulsion in situ gel. Asian J Pharm Clin Res 2019;12:1-5.

9. Gupta R, Kumar M, Sharma HK. Span 80-tween 80 based fluidfilled organogel for topical delivery of fluconazole. Int J Sci Res Rev 2014;3:29-46.

10. Lin TK, Zhong L, Santiago JL. Anti-inflammatory and skin barrier repair effects of topical application of some plant oils. Int J Mol Sci 2018;19:1-21.

11. Balata GF, Moneom Shamardl H-El. Formulation of chlorpheniramine maleate in span 60/Tween20 based organogels for transdermal delivery. J Innov Pharma Bio Sci 2017;4:49-57.

12. Indian Pharmacopoeia; Government of India, Ministry of Health and Family Welfare Department. 7th edition; 2014. p. 1, 559-621.

13. Shukla AK, Yadav SK, Tiwari V. Linear models for S-shaped growth curves. J Stat Appl Prob 2015;4:113-7.

14. Friction welding of dissimilar plastic-based material by metal powder reinforcement. Singh R, Kumar R. Reference module in materials science and materials engineering. Hashmi MSJ. Editor. Dublin, Ireland; 2017. p. 1-16.

15. Bartosova L, Bajgar J. Transdermal drug delivery in vitro using diffusion cells. Curr Med Chem 2012;19:4671-7.

16. Organogels in controlled drug delivery. Singh VK, Behera B, Sagiri SS, Pal K, Anis A, Bhattacharya MK. Handbook of encapsulation and controlled release: Mishra M. Editor. London, UK: CRC Press; 2015. p. 1035-66.

17. Dautel OJ, Robitzer M, Lere Porte JP, Serein Spirau F, Moreau JJ. Selforganized ureido substituted diacetylenic organogel. Photopolymerization of one-dimensional supramolecular assemblies to give conjugated nafiber s. J Am Chem Soc 2006;128:16213-23.

18. Li Y, Wang T, Liu M. Ultrasound induced formation of organogel from a glutamic dendron. Tetrahedron 2007;63:7468-73.

19. Murdan S, Gregoriadis G, Florence AT. Interaction of a nonionic surfactant-based organogel with aqueous media. Int J Pharm 1999;180:211-4. 